\title{
Resultados Tardios de Pacientes Submetidos a Implante de Stents Farmacológicos do Registro SAFIRA
}

\author{
Daniel de Oliveira Neto Barbosa, Julio Flavio Meirelles Marchini, Mohamad Said Ghandour, \\ Bruno Veras Bezerra, Salvador André Bavaresco Cristovão, Nadia Mendonça Carnieto, \\ Eduardo Erudilho, Adnan Ali Salman, Maria Fernanda Zuliani Mauro, José Armando Mangione
}

\begin{abstract}
RESUMO
Introdução: A doença arterial coronária continua sendo um dos maiores problemas de saúde pública da atualidade e a intervenção coronária percutânea com stents farmacológicos é a forma mais frequente de tratamento desses pacientes. O objetivo deste estudo foi avaliar a taxa de desfechos clínicos no seguimento clínico de até 10 anos dos pacientes tratados com stents farmacológicos. Métodos: Pacientes com indicação de ICP foram incluídos de forma prospectiva, independentemente do quadro clínico ou angiográfico de apresentação. O desfecho primário consistiu na avaliação da taxa de eventos cardíacos adversos maiores combinados (óbito cardíaco, infarto agudo do miocárdio não fatal ou necessidade de revascularização do vaso alvo guiada por isquemia) no seguimento clínico tardio. Resultados: No período 2002 a 2012, incluímos 1.632 pacientes, com idade de $64,3 \pm 10,9$ anos, $71,4 \%$ eram do sexo masculino e $31,2 \%$ portadores de diabetes mellitus. As taxas de sucesso angiográfico e clínico foram de 99,1\% e $96,7 \%$, respectivamente. O seguimento clínico foi realizado em $95,8 \%$ dos elegíveis em um tempo médio de seguimento de 3,4 $\pm 2,6$ anos. O desfecho primário ocorreu em 246 pacientes $(15,9 \%)$, com taxas de óbito cardíaco de $6,4 \%$, infarto do miocárdio de $3,6 \%$ e revascularização do vaso alvo de $5,5 \%$. A trombose do stent foi verificada em 40 pacientes $(2,4 \%)$. Conclusões: Nesta experiência da prática clínica diária, a utilização dos stents farmacológicos demonstrou resultados clínicos favoráveis agudos e no longo prazo no tratamento de uma população não selecionada de pacientes com doença arterial coronária e graus variáveis de complexidade clínica e angiográfica.
\end{abstract}

DESCRITORES: Doença da artéria coronariana. Intervenção coronária percutânea. Stents farmacológicos. Trombose coronária.

\section{ABSTRACT}

Late Outcomes of Patients Undergoing Drug-Eluting Stent Implantation - The SAFIRA Registry

Background: Coronary artery disease remains a major public healthcare problem worldwide and percutaneous coronary intervention with drug-eluting stents is the most frequent treatment option for these patients. The objective of this study was to evaluate the rate of clinical events in up to 10 years of clinical follow-up of patients treated with drug-eluting stents. Methods: We prospectively enrolled patients with an indication for percutaneous coronary intervention despite their clinical or angiographic presentation. The primary endpoint consisted of the evaluation of the composite rate of major adverse cardiac events (cardiac death, non-fatal acute myocardial infarction or the need of ischemia guided target vessel revascularization) in the late clinical follow-up. Results: From 2002 to 2012, we included 1,632 patients with mean age of $64.3 \pm 10.9$ years, $71.4 \%$ were male and $31.2 \%$ had diabetes mellitus. The angiographic and clinical success rates were $99.1 \%$ and $96.7 \%$, respectively. Follow-up was obtained in $95.8 \%$ of the eligible patients at a mean of $3.4 \pm 2.6$ years. The primary endpoint was observed in 246 patients $(15.9 \%)$, with rates of cardiac death of $6.4 \%$, myocardial infarction of $3.6 \%$ and target vessel revascularization of $5.5 \%$. Stent thrombosis was observed in 40 patients $(2.4 \%)$. Conclusions: In this clinical practice experience the use of drug-eluting stents demonstrated favorable acute and long-term results in the treatment of a non-selected population of patients with coronary artery disease and variable degrees of clinical and angiographic complexity.

DESCRIPTORS: Coronary artery disease. Percutaneous coronary intervention. Drug-eluting stents. Coronary thrombosis. 
D entre as doenças cardiovasculares, a doença arterial coronária (DAC) continua sendo um dos maiores problemas de saúde pública em todo o mundo. Estima-se que, nos Estados Unidos, um em cada três adultos, ou seja, 81 milhões de pessoas, apresenta alguma forma de doença cardiovascular, incluindo mais de 17 milhões com DAC e que, dentre eles, aproximadamente 10 milhões tenham angina de peito. ${ }^{1,2}$

A intervenção coronária percutânea (ICP), que apresentou expressiva evolução tecnológica ao longo dos anos, culminando com o advento dos stents farmacológicos (SF) é, atualmente, a técnica mais utilizada no tratamento da DAC. ${ }^{3-8}$ Inicialmente empregada em lesões de baixa complexidade clínica e angiográfica, observou-se, gradativamente, expansão de suas indicações para lesões e pacientes mais complexos, obtendo-se resultados clínicos consistentemente superiores aos ICP com stents convencionais. ${ }^{9}$

Entretanto, existem poucos estudos que avaliam o seguimento de longo prazo dessa tecnologia ${ }^{10,11}$ e, em especial, são escassos os dados oriundos da população brasileira, ${ }^{12}$ cujas características culturais e sociais próprias influenciam a aderência ao tratamento e, também, por apresentarem composição genética diferente da de populações dos grandes registros oriundos dos Estados Unidos e Europa. ${ }^{13,14}$

Nosso objetivo foi avaliar os resultados tardios (em até 10 anos) de uma coorte não selecionada de pacientes submetidos a implante de SF em um hospital terciário do Estado de São Paulo.

\section{MÉTODOS}

Trata-se de um estudo prospectivo, não randomizado, de pacientes consecutivos portadores de DAC, tratados com pelo menos um SF, no período de julho de 2002 a agosto de 2012, no Complexo Hospitalar da Real e Benemérita Associação Portuguesa de Beneficência, que inclui os Hospitais São Joaquim e São José, do Serviço Arie Cardiologia Intervencionista. O protocolo deste projeto foi devidamente aprovado pelo Comitê de Ética em Pesquisa, sob o número 778-12, e o termo de consentimento livre e esclarecido foi assinado.

Foram incluídos pacientes com indicação de revascularização coronária percutânea, independentemente do quadro clínico ou angiográfico, passíveis de serem tratados com os SF disponíveis no setor de material especial do hospital. Excluímos pacientes que apresentassem contraindicação ao uso de antiplaquetários ou de anticoagulantes previstos pelo protocolo medicamentoso, ou pacientes que se recusassem a assinar o termo de consentimento livre e esclarecido.

\section{Terapêutica antiagregante plaquetária}

O protocolo incluiu a combinação de dois agentes antiplaquetários, o ácido acetilsalicílico (AAS) e o clopidogrel, nas doses de ataque de 200 e 300 mg, respectivamente, administradas 24 horas antes do procedimento nos casos eletivos. Na síndrome coronária aguda (SCA), nos pacientes que não tinham sido pré-medicados, o AAS foi mastigado na dose de $300 \mathrm{mg}$ e utilizou-se a dose de ataque de $600 \mathrm{mg}$ de clopidogrel. Após o surgimento do ticagrelor e do prasugrel, essas medicações foram incorporadas para uso na SCA ou nos casos eletivos de maior complexidade angiográfica, quando a ICP era realizada ad hoc, nas doses de ataque de $180 \mathrm{mg}$ e $60 \mathrm{mg}$, respectivamente. A utilização dos inibidores da glicoproteína IIb/IIla ficou a critério do operador.

Após o procedimento, o AAS foi mantido indefinidamente na dose de $100 \mathrm{mg} /$ dia. Os inibidores P2Y12 clopidogrel (75 mg/dia), ticagrelor (90 mg, duas vezes ao dia) ou prasugrel (10 mg/dia) foram recomendados por um período mínimo de 1 ano.

\section{Coleta de dados e seguimento clínico}

Os dados foram coletados por meio de formulário específico da equipe e armazenados em um banco de dados especialmente dedicado ao Registro SAFIRA (SegurançA e eFIcácia dos stents farmacológicos em uma população do mundo ReAl). Os pacientes foram seguidos após a alta hospitalar por contato telefônico realizado por um profissional da área da saúde ou por consulta médica, sendo essas avaliações efetuadas com 1, 6 e 12 meses e, então, anualmente.

\section{Técnica do procedimento}

Após a obtenção da via de acesso, a heparina não fracionada foi administrada na dose de $100 \mathrm{U} / \mathrm{kg}$. Quando se utilizaram os inibidores da glicoproteína Ilb/IIla, a dosagem inicial foi de $70 \mathrm{U} / \mathrm{kg}$, procurando-se manter o tempo de coagulação ativado entre 200 a 250 segundos.

As análises angiográficas seriadas foram realizadas pré e pós-procedimento, incluindo-se avaliações qualitativas e quantitativas das lesões coronárias. A aquisição dos angiogramas ocorreu após a administração de mononitrato de isossorbida $10 \mathrm{mg}$ intra-arterial (a não ser que isso fosse clinicamente contraindicado) e incluiu duas projeções ortogonais, separadas por, pelo menos, $30^{\circ}$, procurando evitar a superposição das artérias e otimizando a visualização da lesão alvo.

O procedimento foi monitorado por análise visual e pela angiografia coronária quantitativa, quando necessário, utilizando-se o programa CAAS 2000 (PIE Medical's ${ }^{\circledR}$, Maastricht, Holanda) com técnicos treinados. Os exames foram gravados na velocidade de 15 quadros por segundo em filmes $35 \mathrm{~mm}$ ou no formato digital $\mathrm{DICOM}^{\circledR}$ (Digital Imaging and Communication in Medicine $\left.^{\circledR}\right)$ e arquivados em mídia tipo CDR.

A localização da lesão alvo na árvore coronária foi definida de acordo com o segmento coronário envol- 
vido: ostial, proximal, médio e distal, sendo utilizado o sistema de mapeamento coronário CASS (Coronary Artery Surgery Study) modificado para o estudo BARI (Bypass Angioplasty Revascularization Investigation). ${ }^{15}$

Durante o implante do SF, existiam os objetivos de efetuar a cobertura total da lesão tratada, evitar lesão das bordas dos stents na pós-dilatação e, no caso de implante de dois stents sequenciais, não deixar espaço entre os dispositivos, fazendo-se sobreposição de 2 a $3 \mathrm{~mm}$ das hastes.

\section{Definições}

O sucesso angiográfico foi definido como o implante do stent na lesão alvo com estenose residual $<20 \%$, com fluxo final TIMI III, na ausência de trombos e dissecção arterial. O sucesso clínico foi caracterizado como a obtenção do sucesso angiográfico associado à ausência de eventos clínicos adversos maiores na fase hospitalar.

A trombose do stent foi considerada como qualquer evento isquêmico relacionado à oclusão trombótica do stent, classificada em definitiva, provável e possível e, quanto à evolução temporal, em aguda, subaguda, tardia e muito tardia, de acordo com a definição da Academic Research Consortium (ARC). ${ }^{16}$

Todos os óbitos foram considerados cardíacos, a não ser que uma causa não cardíaca pudesse ser claramente estabelecida. O infarto agudo do miocárdio (IAM) foi definido como aumento da CKMB acima de três vezes o valor de referência, com ou sem o surgimento de novas ondas Q com duração > 0,04 segundo, em duas ou mais derivações contíguas no eletrocardiograma. A cirurgia de revascularização miocárdica de urgência foi qualificada como cirurgia não planejada realizada na fase hospitalar. A revascularização da lesão alvo foi definida como reintervenção em razão da recorrência da lesão dentro do stent ou no segmento, que incluía os $5 \mathrm{~mm}$ proximais ou distais à prótese, e a revascularização do vaso alvo foi caracterizada como reintervenção percutânea ou cirúrgica do vaso alvo.

Complicações por sangramento foram classificadas em maiores e menores, de acordo com a classificação TIMI. ${ }^{17}$ As complicações vasculares incluíram pseudoaneurismas, hematomas, fístulas arteriovenosas, sangramento externo, hematoma retroperitoneal, trombose arterial, abscesso no local da punção e aneurisma micótico.

\section{Desfechos do estudo}

O desfecho primário consistiu na avaliação da taxa de eventos cardíacos adversos maiores (ECAM) combinados (óbito cardíaco, IAM não fatal ou necessidade de revascularização do vaso alvo guiada por isquemia) no seguimento clínico. Os desfechos secundários incluíram a avaliação dos componentes isolados do desfecho primário, o sucesso do procedimento, as complicações vasculares e por sangramento, a trombose de stent, a probabilidade da sobrevivência livre de ECAM em 10 anos e a identificação dos preditores de ECAM, de seus componentes individuais e da trombose dos stents.

\section{Análise estatística}

As variáveis contínuas foram apresentadas como média e desvio padrão, e as categóricas como frequências (número e porcentagem). As curvas de sobrevivência livre de eventos acumulados foram estimadas pelo método de Kaplan-Meier.

Para determinar os preditores independentes de ECAM, de seus componentes e da trombose do stent, foi utilizado o modelo de regressão logística multivariado de Cox, incluindo as variáveis cujos valores de $p$ foram $\leq 15 \%$ no modelo univariado. Os resultados foram apresentados com a razão de risco e o intervalo de confiança de $95 \%$. A premissa de riscos proporcionais foi confirmada testando resíduos de Schoenfeld e não foram encontradas violações.

As análises foram realizadas pelo programa estatístico STATA ${ }^{\circledR}$ (StataCorp Company, College Station, Texas, Estados Unidos), versão 12.

\section{RESULTADOS}

No período de julho de 2002 a agosto de 2012, foram incluídos 1.632 pacientes, com média de idades de $64,3 \pm 10,9$ anos, sendo $71,4 \%$ dos pacientes do sexo masculino e $31,2 \%$ portadores de diabetes mellitus $-9,9 \%$ destes em uso de insulina (Tabela 1 ).

TABELA 1

Características clínicas

\begin{tabular}{lc}
\hline Característica & $\mathbf{n}=\mathbf{1 . 6 3 2}$ \\
\hline Idade, anos & $64,3 \pm 10,9$ \\
Sexo masculino, n (\%) & $1.166(71,4)$ \\
Hipertensão arterial, n (\%) & $1.308(80,1)$ \\
Diabetes mellitus, n (\%) & $510(31,2)$ \\
$\quad$ Em uso de insulina & $161(9,9)$ \\
Dislipidemia, n (\%) & $1.091(66,9)$ \\
Tabagismo, n (\%) & $279(17,1)$ \\
História familiar de doença arterial coronariana, n (\%) & $662(40,6)$ \\
Infarto do miocárdio prévio, n (\%) & $391(23,9)$ \\
Intervenção coronária percutânea prévia, n (\%) & $316(19,4)$ \\
Revascularização do miocárdio prévia, n (\%) & $250(15,3)$ \\
Doença pulmonar obstrutiva crônica, n (\%) & $21(1,3)$ \\
Doença vascular periférica, $\mathrm{n}(\%)$ & $45(2,8)$ \\
Insuficiência renal crônica, n (\%) & $130(8)$ \\
Fração de ejeção do ventrículo esquerdo < 40\%, n (\%) & $238(14,6)$ \\
\hline
\end{tabular}


Com relação ao quadro clínico, 872 (53,4\%) eram portadores de angina estável, 373 (22,9\%) eram assintomáticos $-87,1 \%$ tinham provas isquêmicas positivas, $366(22,4 \%)$ apresentavam-se em SCA sem elevação ST e $21(1,3 \%)$ em SCA com elevação de ST. Dos pacientes com SCA sem elevação ST, 197 (54\%) foram classificados como de alto risco, 118 (32\%) de risco moderado e 51 (14\%) de baixo risco.

\section{Características angiográficas e do procedimento}

A maioria da população foi composta por pacientes com acometimento multiarterial $(61,4 \%)$, e o vaso mais frequentemente tratado foi a artéria descendente anterior $(63,3 \%)$. A intervenção no tronco da coronária esquerda foi realizada em 83 casos $(3,4 \%)$ e, destes, 17 (1\%) não eram protegidos por revascularização cirúrgica prévia. As lesões de maior complexidade tipo B2 e C estiveram presentes em 1.639 (63,2\%) dos casos (Tabela 2).

A via de acesso mais utilizada foi a femoral, em 973 casos (58\%). O emprego da técnica radial iniciou-se em 2006, sendo efetuada em 699 procedimentos (41\%) e, a partir de 2009, tornou-se a via de acesso preferencial do serviço. A via braquial foi empregada em 17 casos (1\%). A Figura 1 mostra a taxa de utilização das vias femoral e radial ao longo do tempo. Foram realizados 1.689 procedimentos, com duração de 8 a 300 minutos (média de 47,8 \pm 2,3 minutos) e o volume médio de contraste utilizado foi de $138 \pm 63,8 \mathrm{~mL}$, variando de 30 a $350 \mathrm{~mL}$. Foram tratadas 2.592 lesões, utilizando-se 2.664 SF, com média de 1,03 stent/lesão. Em 963 lesões $(37,1 \%)$, efetuou-se o implante direto do stent, sem a necessidade de pré-dilatação.

O diâmetro médio dos stents utilizados foi de $2,98 \pm 1,25 \mathrm{~mm}$, com comprimento médio de 18,8 \pm $8,0 \mathrm{~mm}$, e a pressão final média de liberação foi de $14,8 \pm 2,7 \mathrm{~atm}$

\section{Resultados na fase hospitalar}

O sucesso dos procedimentos angiográfico e clínico foi de $99,1 \%$ e 96,7\%, respectivamente. O desfecho combinado de óbito, IAM não fatal e revascularização de urgência ocorreu em $40(2,4 \%)$ casos, óbito em 8 $(0,5 \%)$, IAM em $29(1,8 \%)$ e a necessidade de revascularização de urgência em 3 pacientes $(0,2 \%)$.

Sangramento maior foi verificado em 21 procedimentos $(1,2 \%)$, sendo $12(0,7 \%)$ hematomas $\geq 5 \mathrm{~cm}$ e $9(0,5 \%)$ casos de queda da hemoglobina $\geq 5 \mathrm{~g} / \mathrm{dL}$. O sangramento menor ocorreu em 26 (1,5\%) casos, devido a $21(1,2 \%)$ hematomas $<5 \mathrm{~cm}$, e em $5(0,3 \%)$ pacientes observou-se queda da hemoglobina $<5 \mathrm{~g} / \mathrm{dL}$.

As complicações vasculares ocorreram em 60 (3,5\%) procedimentos, somando-se a eles mais 13 casos de fístula arteriovenosa $(0,8 \%)$.

O tempo de internação hospitalar variou de 1 a 32 dias, com média de 1,5 \pm 1,4 dia.
TABELA 2

Características angiográficas e do procedimento

\begin{tabular}{|c|c|}
\hline Características & $\begin{array}{c}n=1.689 \text { procedimentos } / \\
2.592 \text { lesões }\end{array}$ \\
\hline \multicolumn{2}{|l|}{$\begin{array}{l}\text { Extensão da doença } \\
\text { arterial coronária, } \mathrm{n}(\%)\end{array}$} \\
\hline Uniarterial & $630(38,6)$ \\
\hline Biarterial & $556(34,1)$ \\
\hline Triarterial & $446(27,3)$ \\
\hline \multicolumn{2}{|l|}{ Vaso tratado, n (\%) } \\
\hline Descendente anterior & $1.067(63,3)$ \\
\hline Coronária direita & $680(40,2)$ \\
\hline Circunflexa & $618(36,6)$ \\
\hline Tronco da coronária esquerda & $88(5,2)$ \\
\hline $\begin{array}{l}\text { Tronco da coronária esquerda } \\
\text { não protegido }\end{array}$ & $17(1,0)$ \\
\hline Enxerto venoso/arterial & $90(5,2)$ \\
\hline \multicolumn{2}{|l|}{ Características da lesão, n (\%) } \\
\hline Presença de trombo & $71(2,7)$ \\
\hline Calcificação moderada/importante & $181(6,9)$ \\
\hline Reestenose & $124(4,8)$ \\
\hline Bifurcação & $166(6,4)$ \\
\hline \multicolumn{2}{|l|}{ Tipos de stents utilizados, $\mathrm{n}(\%)$} \\
\hline Endeavor ${ }^{\circledR}$ Sprint & $730(27,4)$ \\
\hline Cypher ${ }^{\circledR}$ & $525(19,7)$ \\
\hline Taxus $^{\circledR}$ & $405(15,2)$ \\
\hline Xience V ${ }^{\circledast} /$ Promus & $386(14,5)$ \\
\hline BioMatrix ${ }^{\circledR}$ & $178(6,7)$ \\
\hline Promus Element ${ }^{\circledR}$ & $146(5,5)$ \\
\hline Outros & $294(11,0)$ \\
\hline Implante direto, n (\%) & $963(37,1)$ \\
\hline Pressão final de liberação, mmHg & $14,8 \pm 2,7$ \\
\hline Inibidores da glicoproteína llb/Illa, n (\%) & $21(1,2)$ \\
\hline Extensão da lesão, mm & $20,1 \pm 6,9$ \\
\hline Diâmetro de referência do vaso, mm & $2,76 \pm 0,68$ \\
\hline \multicolumn{2}{|l|}{ Diâmetro mínimo da luz, mm } \\
\hline Pré & $0,54 \pm 0,32$ \\
\hline Pós & $2,88 \pm 0,46$ \\
\hline \multicolumn{2}{|l|}{ Diâmetro da estenose, \% } \\
\hline Pré & $80,3 \pm 10,6$ \\
\hline Pós & $1,0 \pm 4,3$ \\
\hline
\end{tabular}

\section{Resultados tardios}

O seguimento clínico foi realizado em 1.542 pacientes $(95,8 \%$ dos elegíveis). O tempo médio de seguimento foi de $3,4 \pm 2,6$ anos (com variação de 1 mês a 10 anos) (Figura 2). 


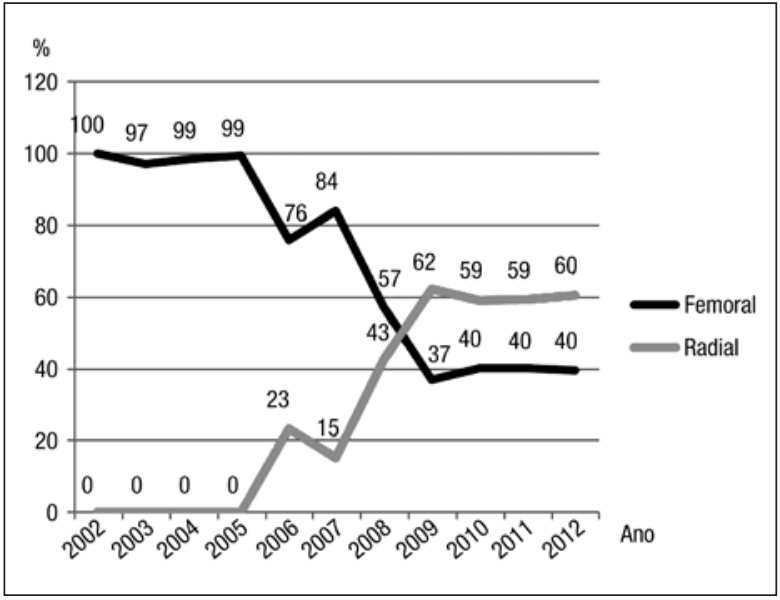

Figura 1. Evolução temporal da utilização das vias radial e femoral.

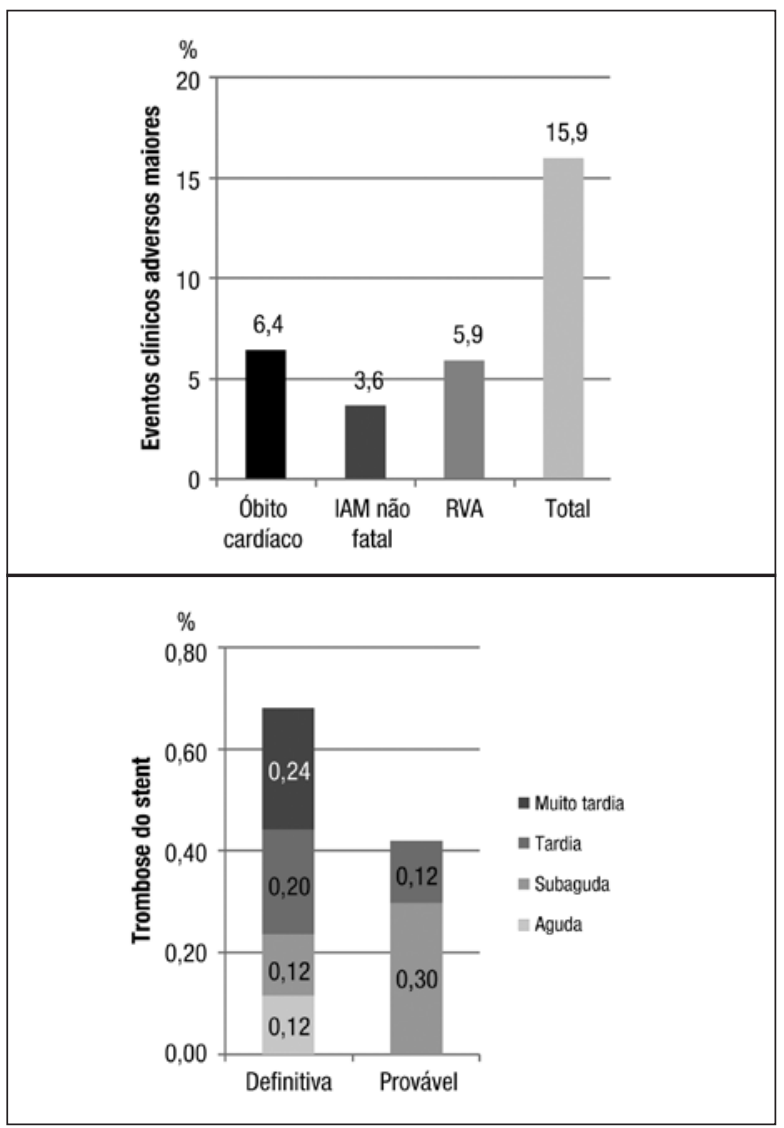

Figura 2. Eventos clínicos adversos maiores e trombose do stent no acompanhamento tardio. IAM: infarto agudo do miocárdio; RVA: revascularização do vaso alvo.

O desfecho combinado de óbito cardíaco, IAM não fatal e revascularização do vaso alvo guiada por isquemia (objetivo primário) ocorreu em 245 pacientes $(15,9 \%)$; o desfecho combinado de óbito cardíaco, IAM não fatal e revascularização da lesão alvo ocorreu em
234 pacientes $(15,1 \%)$. A necessidade de revascularização da lesão alvo ocorreu em 71 pacientes $(4,6 \%)$ e a mortalidade total ocorreu em 142 (9,2\%) casos. Trinta e sete pacientes $(2,4 \%)$ submeteram-se à revascularização cirúrgica do miocárdio e $27(1,7 \%)$ à ICP em outro vaso.

A trombose do stent foi observada em 40 pacientes $(2,4 \%)$ ao longo de todo o período de evolução. A trombose do stent definitiva ou provável ocorreu em 18 pacientes $(1,1 \%)$ com sua distribuição temporal sumarizada na figura 2 .

A figura 3 mostra as curvas de Kaplan-Meier de sobrevivência livre de ECAM, óbito cardíaco, IAM e revascularização do vaso alvo, respectivamente, ao longo de 10 anos de acompanhamento.

Os preditores independentes do desfecho combinado de óbito cardíaco, IAM não fatal e revascularização do vaso alvo guiada por isquemia, de seus componentes isolados e da trombose dos stents, identificados por meio da análise multivariada, são apresentados na Tabela 3.

\section{DISCUSSÃO}

Os resultados deste estudo demonstram que o emprego dos SF para o tratamento da DAC, na prática clínica diária, em pacientes com graus variáveis de complexidade clínica e angiográfica, é um procedimento eficaz e seguro, possibilitando resultados favoráveis nas fases hospitalar e tardia, semelhantes aos verificados nos estudos randomizados iniciais, que testaram os SF.-8

Até onde sabemos, essa é uma, de duas ${ }^{18}$ experiências nacionais publicadas, que apresentou seguimento clínico de até 10 anos, de pacientes não selecionados, tratados com SF.

$\mathrm{Na}$ análise das características clínicas deste estudo, observou-se que a idade média (64,3 \pm 10,9 anos) e o porcentual de pacientes do sexo masculino $(71,4 \%)$ estavam de acordo com estudos sobre SF já publicados, ${ }^{3-8,19-22}$ Chama a atenção o fato de que $31,2 \%$ da população era constituída por portadores de diabetes mellitus, índice este mais elevado que aqueles encontrados em estudos randomizados ${ }^{3-8}$ e em alguns registros, como o Swedish Coronary Angiography and Angioplasty Registry (SCAAR) ${ }^{22}(23,8 \%)$ e o RESEARCH ${ }^{23}$ (22\%); esse valor, entretanto, está de acordo com o da Emilia Romagna $\operatorname{REAL}^{10}(30,7 \%)$.

A ICP em tronco da coronária esquerda não protegido foi realizada somente em 17 pacientes $(1,0 \%)$ da nossa casuística, fato que também foi verificado no registro DESIRE, ${ }^{18}$ que analisou 4.229 pacientes submetidos a implante de SF e mostrou que a lesão de tronco esteve presente em 1,5\% dos casos tratados. Os achados desses dois centros estão de acordo com o banco de dados da Central Nacional de Intervenções Cardiovasculares (CENIC), ${ }^{24}$ no qual foi registrado um total de 67.887 ICP realizadas entre 2009 a 2011, 


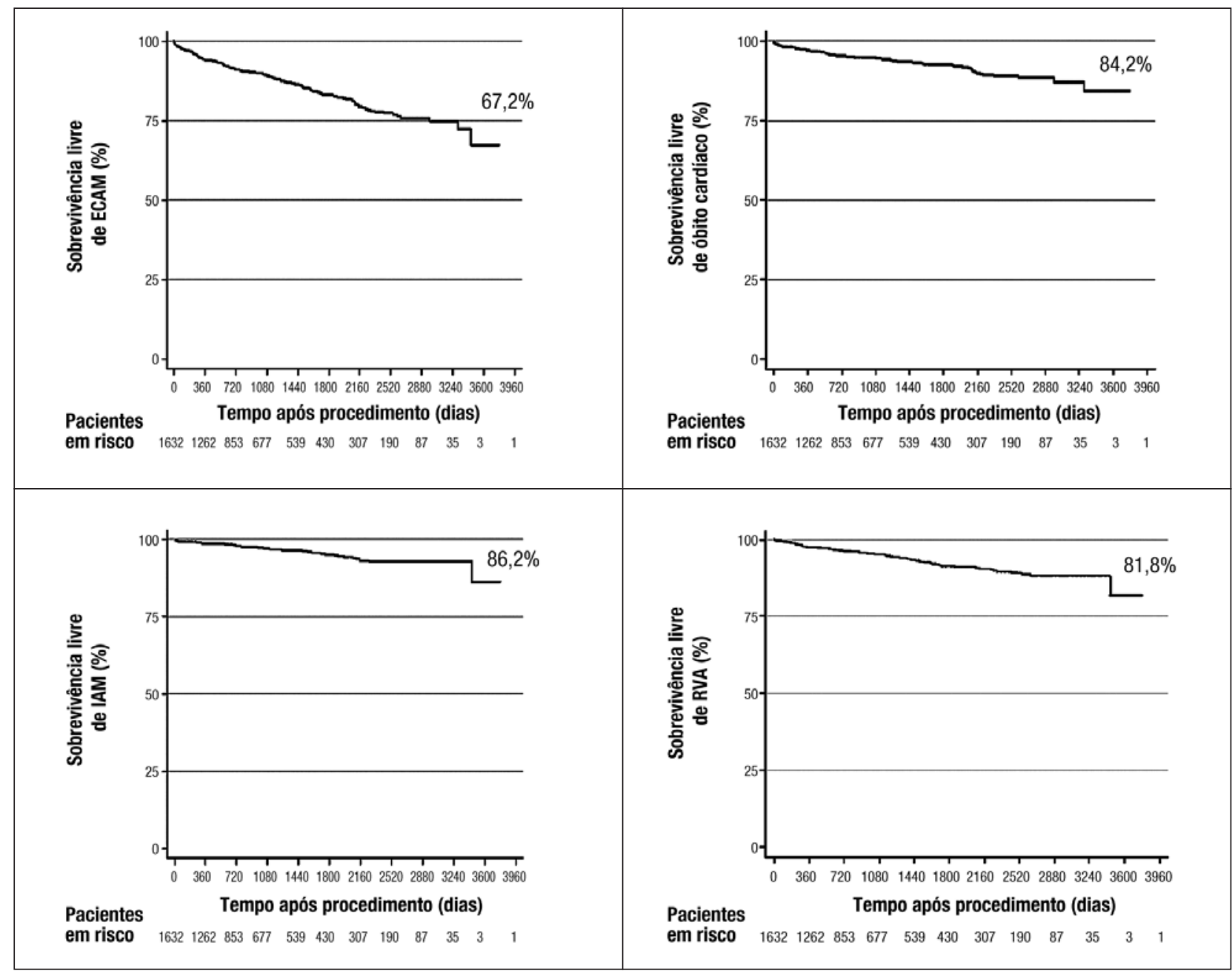

Figura 3. Taxas de sobrevivência livre de eventos cardíacos adversos maiores (ECAM), óbito cardíaco, infarto agudo do miocárdio (IAM) e revascularização do vaso alvo, respectivamente, ao longo de 10 anos de acompanhamento.

sendo que somente $1.081(1,6 \%)$ delas foram realizadas para o tratamento de lesões localizadas no tronco da coronária esquerda, demonstrando que, no Brasil, o tratamento percutâneo dessa situação é ainda reservado para um número restrito de pacientes.

Os resultados hospitalares obtidos em nossa série demonstram que a realização da ICP, na prática clínica atual, é um procedimento seguro e que apresenta alta taxa de sucesso clínico e angiográfico. Na verdade, a utilização dos stents coronários proporcionou melhores resultados imediatos, pois corrigiu algumas importantes limitações da angioplastia coronária com o cateter balão, como a oclusão aguda do vaso e as dissecções da parede arterial. De fato, a cirurgia de urgência, em nosso estudo, foi necessária somente em $0,2 \%$ dos casos.

O sangramento maior ocorreu em 1,2\% dos pacientes, taxa inferior a encontrada com a utilização da técnica femoral $(2,3 \%)$ na meta-análise publicada por Jolly et al., ${ }^{25}$ que analisou 23 estudos randomizados (6 diagnósticos) que compararam as vias radial e femoral. Esse fato reforça os achados sobre o emprego da via radial ser fator importante na redução desse tipo de complicação.

O seguimento clínico de até 10 anos (média de 3,4 \pm 2,6 anos) de nossa população mostrou que o desfecho primário deste estudo (óbito cardíaco, IAM não fatal e revascularização do vaso alvo guiada por isquemia) ocorreu em 15,9\%. Tu et al. ${ }^{26}$ em análise de 3.751 pacientes submetidos a implante de SF realizada na província de Ontário, no Canadá, mostraram, ao final de 2 anos, uma incidência desses eventos de 17,4\%. Em outro importante registro, o REAL, ${ }^{10}$ que contou com 3.064 submetidos a implante de SF, com 2 anos de seguimento, essa taxa foi de 20,2\%.

A trombose total do stent verificada foi de 2,4\% e a classificada como definitiva ou provável foi de $1,1 \%$. No estudo de Marzocchi et al., ${ }^{10}$ a trombose comprovada angiograficamente ocorreu em 1\% dos 
TABELA 3

Preditores independentes de eventos cardíacos adversos maiores (ECAM), óbito, infarto agudo do miocárdio (IAM), revascularização do vaso alvo (RVA) e trombose de stent

\begin{tabular}{|c|c|c|c|}
\hline Variável & Razão de risco & Intervalo de confiança de $95 \%$ & Valor de $p$ \\
\hline \multicolumn{4}{|l|}{ ECAM } \\
\hline Doença vascular periférica & 2,14 & $1,23-3,73$ & 0,01 \\
\hline Insuficiência renal crônica & 2,01 & $1,32-3,07$ & 0,01 \\
\hline Tronco de coronária esquerda & 1,99 & $1,04-3,79$ & 0,04 \\
\hline Ponte de veia safena & 1,96 & $1,14-3,35$ & 0,01 \\
\hline IAM prévio & 1,51 & $1,10-2,06$ & 0,01 \\
\hline Multiarterial & 1,45 & $1,06-1,98$ & 0,02 \\
\hline Idade & 1,01 & $1,00-1,03$ & 0,02 \\
\hline \multicolumn{4}{|l|}{ Óbito } \\
\hline Insuficiência renal crônica & 3,78 & $2,26-6,34$ & $<0,01$ \\
\hline Ponte de veia safena & 2,47 & $1,21-5,07$ & 0,01 \\
\hline Doença vascular periférica & 2,25 & $1,12-4,41$ & 0,02 \\
\hline Calcificação & 1,87 & $1,23-2,83$ & 0,01 \\
\hline Diabetes mellitus & 1,82 & $1,19-2,76$ & 0,01 \\
\hline Stent Taxus ${ }^{\circledR}$ & 1,60 & $1,03-2,50$ & 0,04 \\
\hline Disfunção de ventrículo esquerdo moderada/grave & 1,41 & $1,11-1,81$ & 0,01 \\
\hline Idade & 1,06 & $1,04-1,08$ & $<0,01$ \\
\hline \multicolumn{4}{|l|}{ IAM } \\
\hline Doença vascular periférica & 3,77 & $1,34-10,60$ & 0,01 \\
\hline Sexo feminino & 2,38 & $1,06-5,34$ & 0,04 \\
\hline \multicolumn{4}{|l|}{ RVA } \\
\hline Tronco de coronária esquerda & 2,88 & $1,17-7,12$ & 0,02 \\
\hline IAM & 1,73 & $1,07-2,78$ & 0,02 \\
\hline \multicolumn{4}{|l|}{ Trombose de stent } \\
\hline Doença vascular periférica & 5,02 & $2,03-12,36$ & $<0,01$ \\
\hline Insuficiência renal crônica & 3,14 & $1,35-7,31$ & $<0,01$ \\
\hline Idade & 1,04 & $1,01-1,07$ & 0,02 \\
\hline
\end{tabular}

pacientes; já Costa Jr. et al. ${ }^{12}$ relataram incidência total de $1,6 \%$ dentre 2.365 pacientes analisados - em 1\%, foi classificada como definitiva.

As curvas de sobrevivência livre de eventos em um período de até 10 anos, em nossa série, mostraram resultados favoráveis com a utilização dos SF na prática clínica diária (ECAM de $67,2 \%$, óbito cardíaco de $84,2 \%$, IAM não fatal de $86,2 \%$ e revascularização do vaso alvo de $81,8 \%$ ). Nos achados de até 5 anos de acompanhamento do registro DESIRE, essas taxas foram de 91,5\% para sobrevivência livre de ECAM e de 96,7\% para a sobrevivência livre de revascularização da lesão alvo. ${ }^{11}$

Pela análise multivariada, identificamos sete preditores independentes de ECAM. Destes, três (doença vascular periférica, insuficiência renal crônica e lesões off-label/ponte de veia safena) também foram mencionados na publicação de Sousa et al. ${ }^{19} \mathrm{em}$ um período de acompanhamento de até 10 anos. Entre os preditores de óbito cardíaco, chama atenção a utilização do stent Taxus $^{\circledR}$, com uma razão de risco de 1,6 ( $\left.p=0,04\right)$. Stettler et al. ${ }^{20}$ publicaram meta-análise envolvendo 38 estudos randomizados com 18.023 pacientes e não demonstraram diferença em relação à mortalidade total entre os stents metálicos e os SF com eluição de sirolimus ou paclitaxel. Entretanto, o stent com eluição de sirolimus apresentou redução relativa de $17 \%$ na taxa de IAM, comparado ao stent com eluição de paclitaxel $(p=0,05)$. A doença vascular periférica também se mostrou preditora de IAM (razão de risco de 3,77 ), achado que também foi verificado na análise de Sousa et al. ${ }^{19}$ Para a revascularização do vaso alvo, identificamos como preditores a lesão localizada 
no tronco da coronária esquerda e os pacientes que apresentaram história de IAM prévio.

Com relação à trombose dos stent, os preditores foram os seguintes: doença vascular periférica, insuficiência renal crônica e idade. A publicação de lakovou et al., ${ }^{21}$ que analisou 2.229 pacientes submetidos a implante de SF em três centros europeus, demonstrou que, além da insuficiência renal crônica evidenciada em nosso estudo, o diabetes mellitus, as lesões localizadas em bifurcação, a descontinuidade prematura da terapia antiagregante plaquetária e a baixa fração de ejeção do ventrículo esquerdo foram preditores independentes da trombose dos stents. Houve, neste estudo, uma tendência a uma maior taxa de trombose com o stent com eluição de paclitaxel, comparado ao stent com sirolimus $(0,8 \%$ vs. $1,7 \% ; p=0,09)$. Este achado está de acordo com o encontrado na meta-análise de Stettler et al. ${ }^{20}$ que observou aumento relativo de $86 \%$ na trombose definitiva tardia com o stent com eluição de paclitaxel, comparado ao stent com sirolimus $(p=0,0041)$.

\section{CONCLUSÕES}

Nesta experiência de pacientes da prática clínica, a utilização dos stents farmacológicos demonstrou resultados clínicos favoráveis agudos e no longo prazo no tratamento de uma população não selecionada de indivíduos com graus variáveis de complexidade clínica e angiográfica. Esses achados, em conjunto com estudos prévios, demonstram a eficácia e a segurança dessa tecnologia no tratamento da doença arterial coronária.

\section{CONFLITO DE INTERESSES}

Não há.

\section{FONTE DE FINANCIAMENTO}

Não há.

\section{REFERÊNCIAS}

1. Mensah GA, Brown DW. An overview of cardiovascular disease burden in the United States. Health Aff (Milwood). 2007;26(1):38-48.

2. Lloyd-Jones D, Adams RJ, Brown TM, Carnethon M, Dai S, De Simone G, et al.; American Heart Association Statistics Committee and Stroke Statistics Committee. Heart disease and stroke statistics - 2010 uptade: a report from the American Heart Association. Circulation. 2010;121(7):e46-e215.

3. Sousa JE, Costa MA, Abizaid A, Feres F, Seixas AC, Tanajura LF, et al. Four-year angiographic and intravascular ultrasound follow-up of patients treated with sirolimus-eluting stents. Circulation. 2005;111(18):2326-9.

4. Sousa JE, Costa MA, Farb A, Abizaid A, Sousa A, Seixas $\mathrm{AC}$, et al. Vascular healing 4 years after the implantation of sirolimus-eluting stent in humans: a histopathological examination. Circulation. 2004;110(1):e5-6.

5. Morice MC, Serruys PW, Sousa JE, Fajadet J, Ban Hayashi E, Perin M.; RAVEL Study Group. Randomized study with the sirolimus-coated Bx Velocity balloon-expandable stent in the treatment of patients with de novo native coronary artery lesions. A randomized comparison of a sirolimus eluting stent with a standard stent for coronary for coronary revascularization. $\mathrm{N}$ Engl J Med. 2002;346(23):1773-80.

6. Moses JW, Leon MB, Popma JJ, Fitzgerald PJ, Holmes DR, O'Shaughnessy C; SIRIUS Investigators. Sirolimus-eluting stents versus standard stents in patients with stenosis in a native coronary artery. N Engl J Med. 2003;349(14):1315-23.

7. Schofer J, Schluter M, Gershlick AH, Wijns W, Garcia E, Schampaert E; E-SIRIUS Investigators. Sirolimus-eluting stents for treatment of patients with long atherosclerotic lesions in small coronary arteries: double-blind, randomised controlled trial (E-SIRIUS). Lancet. 2003;362(9390):1093-9.

8. Schampaert E, Cohen EA, Schluter M, Reeves F, Traboulsi M, Title LM; C-SIRIUS Investigators. The Canadian study of the sirolimus-eluting stent in the treatment of patients with long de novo lesions in small native coronary arteries (C-SIRIUS). J Am Coll Cardiol. 2004;43(6):1110-5.

9. Kirtane AJ, Gupta A, Iyengar S, Moses JW, Leon MB, Applegate $R$, et al. Safety and efficacy of drug-eluting and bare metal stents: comprehensive meta-analysis of randomized trials and observational studies. Circulation. 2009;119(25):3198-206.

10. Marzocchi A, Saia F, Piovaccari G, Manari A, Aurier E, Benassi $A$, et al. Long-term safety and efficacy of drug-eluting stents. Two years results of the REAL (Registro AngiopLastiche dellEmilia Romagna) multicenter registry. Circulation. 2007; 115(25):3181-8.

11. Ellis SG, Stone GW, Cox DA, Hermiller S, O’Shaughnessy C, Mann T, et al. Long-term safety and efficacy with Paclitaxeleluting stents: 5 years final results of the Taxus IV clinical trial (TAXUS IV-SR: treatment of de novo coronary disease using a single Paclitaxel-eluting stent). J Am Coll Cardiol Intev. 2009;2(12):1248-59

12. Costa Jr. JR, Sousa AGMR, Moreira AC, Costa RA, Maldonado G, Cano MN, et al. Impacto do uso rotineiro de stents farmacológicos na prática intervencionista contemporânea de um centro terciário: experiência de uma década do Registro DESIRE. Rev Bras Cardiol Invasiva. 2012;20(4):355-60.

13. Pavao R, Marin-Neto JA, Novaes GC, Pinto MR, Figueiredo GL, Lago IM, et al. Avaliação a médio prazo do controle de fatores de risco do doença cardiovascular em coorte prospectiva de pacientes de alto risco, tratados por intervenção coronária percutânea. Rev Bras Cardiol Invasiva. 2013;21(2):121-7.

14. Giolo SR, Soler JM, Greenway SC, Almeida MA, Andrade M, Seidman JG, et al. Brazilian urban population genetic structure reveals a high degree of admixture. Eur J Hum Genet. 2012; 20(1):111-6.

15. Alderman EL, Stadius M. The angiographic definitions of the Bypass Angioplasty Revascularization Investigation. Coron Artery Dis. 1992;3(12):1189-207.

16. Mehilli J, Byrne RA, Tiroch K, Pinieck S, Schulz S, Kufner $S$, et al.; ISAR-DESIRE 2 Investigators. Randomized trial of paclitaxel- versus sirolimus-eluting stents for treatment of COronary restenosis in sirolimus-eluting stents: the ISAR-DESIRE 2 (Intracoronary Stenting and Angiographic Results: Drug Eluting Stents for In-Stent Restenosis 2) study. J Am Coll Cardiol. 2010;55(24):2710-6.

17. Chesebro JH, Knatterud G, Roberts R, Borer J, Cohen LS, Dalen J, et al. Thrombolysis in Myocardial Infarction (TIMI) Trial, Phase I: a comparison between intravenous tissue plasminogen activator and intravenous streptokinase clinical findings through hospital discharge. Circulation. 1987;76(1):142-54.

18. Sousa A, Costa R, Moreira AC, Cano M, Maldonado G, Costa $\mathrm{RA}$, et al. Long-term clinical outcomes of the drug-eluting stents in the real world (DESIRE) registry. J Interv Cardiol. 2008; 21(4):307-14 
19. Sousa JEMR, Sousa AGMR, Costa Jr. JR, Costa R, Moreira AC, Campos Neto CM, et al. Registro DESIRE: uma década (20022012) de emprego clínico dos stents farmacológicos no tratamento da doença arterial coronária. São Paulo: Atheneu; 2012.

20. Stettler C, Wandel J, Alleman S, Kastrati A, Morice MC, Schomig A, et al. Outcomes associated with drug-eluting and bare-metal stents: a collaboration network meta-analysis. Lancet. 2007;370(9591):937-48.

21. lakovou I, Schmidt T, Bonzzoni E, Ge L, Sangiorgi GM, Goran $\mathrm{S}$, et al. Incidence, predictors and outcome of thrombosis after successful implantation of drug-eluting stents. JAMA. 2005; 293(17);2126-30.

22. Carlsson J, James SK, Lindbäck J, Scherstén F, Nilsson T, Stenestrand U, et al.; SCAAR (Swedish Coronary Angiography and Angioplasty Registry) Study Group. Outcome of drug-eluting stent versus bare metal stenting used according to on- and off-label criteria. J Am Coll Cardiol. 2009;53(16):1389-98.

23. Lemos PA, Hoye A, Goedhart D, Arampatzis CA, Saia F, van der Giessen WJ, et al. Clinical, angiographic, and procedural predictors of angiographic restenosis after sirolimus-eluting stent implantation in complex patients. An evaluation from the rapamycin-eluting stent evaluated at Rotterdam Cardiology Hospital (RESEARCH) Study. Circulation. 2004;109(11): 1366-70.

24. Kirtane AJ, Gupta A, lyenagar S, Moses SW, Leon MB, Applegate $R$, et al. Safety and efficacy of drug-eluting and bare metal stents: comprehensive meta-analysis of randomized trials and observational studies. Circulation. 2009;119(25):3198-206.

25. Jolly SS, Amlani S, Hamon M, Yusuf S, Mehta SR. Radial versus femoral access for coronary angiography or intervention and the impact on major bleeding and ischemic events: a systematic review and meta-analysis of randomized trials. Am Heart J. 2009;157(1):132-40.

26. Tu JV, Bowen J, Chiu M, Ko DT, Austin PC, He Y, et al. Effectiveness and safety of drug-eluting stents in Ontario. N Engl J Med. 2007;357(14):1393-402. 